\title{
Inflammation-associated regulation of the macrophage inhibitory cytokine (MIC-1) gene in prostate cancer
}

\author{
SEEMA DUBEY $^{1}$, PETER VANVELDHUIZEN ${ }^{2,3}$, JEFFREY HOLZBEIERLEIN ${ }^{1}$, \\ OSSAMA TAWFIK ${ }^{4}$, J. BRANTLEY THRASHER ${ }^{1}$ and DEV KARAN ${ }^{1,2}$ \\ ${ }^{1}$ Department of Urology, University of Kansas Medical Center, Kansas City, KS 66160; ${ }^{2}$ Cancer Research Unit, \\ Veterans Affairs Medical Center, Kansas City, MO 64128; Departments of ${ }^{3}$ Internal Medicine \\ and ${ }^{4}$ Pathology and Laboratory Medicine, University of Kansas Medical Center, Kansas City, KS 66160, USA
}

Received November 22, 2011; Accepted February 29, 2012

DOI: $10.3892 / \mathrm{ol} .2012 .635$

\begin{abstract}
Macrophage inhibitory cytokine-1 (MIC-1), also known as prostate-derived factor (PDF), is a molecule of the TGF- $\beta$ superfamily and has been associated with the progression of various types of diseases including prostate cancer. Initially identified from activated macrophages, the $M I C-1$ gene may provide a potential link between inflammation and prostate cancer. In this context, we performed $M I C-1$ expression analysis using mouse prostate tissues to determine whether there was any correlation with age and inflammation. Reverse transcription PCR analysis on RNA samples isolated from prostate lobes from prostate-specific antigen transgenic mice of varying ages revealed that $M I C-1$ gene expression is extremely low to non-detectable in the prostate tissues obtained from young mice, while its expression increases in the prostate tissues harvested from elderly mice. Increased $M I C-1$ gene expression in the mouse prostate was found to be associated with an increased level of infiltrating lymphocytes. To confirm this observation, we showed that inflammation-associated cytokines (IL-1 $\beta$ and TNF- $\alpha$ ) significantly upregulate the secretion of the MIC-1 protein in a human prostate cancer cell line (LNCaP cells), while cytokines IL-6 and granulocyte macrophage colony-stimulating factor were less effective. Taken together, these data indicated that inflammation-associated cytokines may play a critical role in the functional regulation of the $M I C-1$ gene in the early stages of prostate cancer development. More studies are required to understand the biological activity of $M I C-1$ gene regulation in the development and progression of prostate cancer.
\end{abstract}

Correspondence to: Dr Dev Karan, Department of Urology, MS 3016, University of Kansas Medical Center, 3901 Rainbow Boulevard, Kansas City, KS 66160, USA

E-mail: dkaran@kumc.edu

Key words: prostate cancer, inflammation, macrophage inhibitory cytokine-1

\section{Introduction}

Prostate cancer is the most common type of cancer in men in Western countries, accounting for one-third of all male cancer diagnoses and $11 \%$ of all mortalities due to cancer (1). Epidemiological and screening studies performed over the past several decades have raised important questions regarding the pathogenesis of prostate cancer, but a definitive cause for this disease has not been established. Although family history and ethnicity are critical risk factors, the diagnosis of prostate cancer is primarily associated with increasing age $(2,3)$. Several epidemiological studies support the association between inflammation and prostate cancer risk $(4,5)$.

Previous studies have suggested that inflammation may be important in the pathogenesis of prostate cancer by altering the tumor environment $(6,7)$. The production of cyclooxygenase (COX) enzymes due to inflammation caused by precancerous tissues provides significant evidence of the role played by inflammation in the development of cancer (8). The inflammatory process is driven by the interactions between various types of cells, proteins and cytokines within the tumor environment $(4,7)$. Various inflammatory cytokines are important mediators of inflammation and are produced by activated macrophages and other immune cells. One of these cytokines, macrophage inhibitory cytokine-1 (MIC-1) was first isolated based on an increased mRNA expression in activated macrophages (9).

MIC-1, also known as growth differentiation factor-15 (GDF-15) or prostate-derived factor (PDF), is a member of the TGF- $\beta$ superfamily and its increased expression has been associated with a variety of cells including breast, gastric and colorectal cancer cells $(10,11)$. However, MIC-1 is significant due to its increased association with high-grade prostate tumors. Protein profiling on micro-dissected samples of matched normal prostate tissue, high-grade prostatic intraepithelial neoplasia (hPIN) and prostate cancer revealed MIC-1 expression in hPIN and cancer cells but not in normal cells, suggesting a potential role of MIC-1 in the pathogenesis of prostate cancer $(12,13)$. Elevated serum MIC-1 levels are associated with a number of disease conditions including chronic inflammatory pathways, and as a predictor of miscarriage in pregnant women (14-16). Specifically in the prostate, 
increasing serum MIC-1 levels are associated with the progression of metastatic prostate cancer $(13,17-19)$. Despite its association with multiple disease conditions, the basic concept of $M I C-1$ gene regulation in prostate cancer development and progression remains largely unknown. To understand the functional regulation of MIC-1, we previously proposed that the MIC-1 gene provides a potential link between inflammation and prostate cancer (20). In this study, using prostate cancer as a model, we studied the impact of inflammation-associated cytokines on MIC-1 expression.

\section{Materials and methods}

Mouse prostate tissue collection and tumor cell lines. Prostate tissue samples from different age groups of male prostatespecific antigen transgenic (PSA-Tg) mice were harvested. These tissue samples were fixed in formalin and/or RNAlater (Ambion, Austin, TX, USA), and stored at $-80^{\circ} \mathrm{C}$. The PSA-Tg mice were a gift from Dr David Lubaroff at the University of Iowa, USA. Prior to prostate tissue harvesting, mice were sacrificed in accordance with guidelines and regulations approved by the Institutional Animal Care and Use Committee. A human prostate cancer cell line (LNCaP cells) was purchased from ATCC (Maryland, MD, USA). The LNCaP cell line was maintained in regular culture medium RPMI-1640 supplemented with $10 \%$ fetal bovine serum (FBS), $1 \%$ glutamine, $1 \%$ sodium pyruvate (Invitrogen, Carlsbad, CA, USA) and $0.05 \mathrm{mg} / \mathrm{ml}$ of gentamicin (Mediatech, Manassas, VA, USA).

Histopathology. Tissue blocks containing the most representative and well-preserved tissue areas were selected for histopathology. Sections $(5 \mu \mathrm{m})$ were cut and stained for hematoxylin and eosin (H\&E) by the core facility at the University of Kansas Medical Center. The H\&E-stained slides from at least three mice per age group were analyzed by the pathologist. Histopathological evaluation included the examination of glandular architecture, nuclear and cellular degenerative changes and the intensity of inflammation along with the presence or absence of premalignant and malignant changes.

$R N A$ isolation and reverse transcription PCR (RT-PCR). Total RNA was extracted from the prostate tissue samples recovered from the RNAlater as well as LNCaP cells using a Qiagen kit as per the manufacturer's instructions. Quantization of RNA was analyzed using a spectrophotometer. Total RNA $(\sim 2 \mu \mathrm{g})$ from the tissue samples and the LNCaP cells was reversetranscribed (RT) using Superscript II (Invitrogen). RT samples were subjected to PCR amplification in a total volume of $50 \mu \mathrm{l}$ containing a mixture of PCR buffer, $\mathrm{MgCl}_{2}, \mathrm{dNTPs}$, each set of primers, and Taq DNA polymerase as per standard protocol (Invitrogen). Oligonucleotide primers for the $M I C-1$ gene were synthesized from the previously published sequences (Accession number AF019770 for the human sequence and NM_011819 for the mouse sequence). GAPDH primers were designed to amplify the product with both mouse and human compatibility. The reaction mixture was denatured at $94^{\circ} \mathrm{C}$ for $2 \mathrm{~min}$ followed by 30 cycles at $94^{\circ} \mathrm{C}$ for $30 \mathrm{sec}, 57^{\circ} \mathrm{C}$ for $45 \mathrm{sec}$, and $72^{\circ} \mathrm{C}$ for $1 \mathrm{~min}$ with a final elongation at $72^{\circ} \mathrm{C}$ for 10 min using an MJ Mini Thermal Cycler (Bio-Rad, Hercules, CA, USA). The amplified PCR products were resolved electrophoretically on an agarose gel stained with ethidium bromide to verify the size of the amplified product.

Western blot for MIC-1 protein analysis. LNCaP cells were plated in a six-well plate at a density of $2 \times 10^{5}$ cells per well under normal culture conditions. Two days later, the medium was replaced with fresh medium, and the cells were cultured for an additional day. Subsequently, the cells were washed with RPMI-1640 phenol red-free medium (without FBS) and starved for $4 \mathrm{~h}$. The cells were then incubated overnight $(\sim 16 \mathrm{~h})$ with RPMI-1640 phenol red-free medium ( $2 \mathrm{ml}$ per well) containing various cytokines at a concentration of $100 \mathrm{ng} / \mathrm{ml}$ (PeproTech, Rocky Hill, NJ, USA). Following incubation, the culture supernatant was collected for quantification of MIC-1 secretion and cells were lysed using RIPA buffer from Sigma (St. Louis, MO, USA).

Approximately $20 \mu \mathrm{l}$ of culture supernatant containing MIC-1 protein was resolved on a ReadyGel and was transferred onto a PVDF membrane (Bio-Rad). Following blocking with $3 \%$ non-fat dry milk in phosphate-buffered saline containing $0.1 \%$ Tween-20 (PBST) for $2 \mathrm{~h}$ at room temperature, the blots were incubated with anti-human MIC-1 primary antibody (1:500 dilution in PBST) overnight at $4^{\circ} \mathrm{C}$. Following repeated washing with PBST, the blots were incubated with corresponding secondary antibody rabbit polyclonal IgG (Abcam, Cambridge, MA, USA) in PBST with 3\% milk for $2 \mathrm{~h}$ at room temperature. Following repeated washing with PBST, the blots were developed using western blotting luminol reagent (Santa Cruz Biotechnology Inc., Santa Cruz, CA, USA), and were exposed to BioMax Film (Kodak), which was developed to analyze the specific band size.

\section{Results and Discussion}

Prostate cancer is the second most common cause of mortality from cancer for men of all ages. However, it is rare in men aged younger than 40 years. Its incidence is known to increase with age with higher percentages of men developing prostate cancer as they become older. Studies $(4,5)$ have suggested that prostate cancer associated with increasing age is also associated with increased susceptibility of the prostate tissue to injury as well as increased predisposition to infections and other insults leading to an increase in inflammatory response. Therefore, to examine the role of MIC-1 in association with inflammation, we analyzed the expression of $M I C-1$ gene in prostate tissue collected from mice of different age groups. For this purpose, we selected the PSA-Tg mouse based on the following criteria: i) that PSA production is confined to the prostate through the use of the probasin promoter, thus simulating the same conditions described in studies on the human prostate; and ii) to investigate the biological process of $M I C-1$ gene regulation in the presence of self-antigen.

MIC-1 association with increasing age in the mouse prostate. Different prostate lobes (ventral, dorsolateral and anterior prostate lobes) from the naïve PSA-Tg mice of different age groups were harvested to prepare total RNA, and were subjected to RT-PCR using primer sets for $M I C-1$ gene expression. The anticipated PCR product of $238 \mathrm{bp}$ was resolved by agarose gel electrophoresis (Fig. 1). In agreement with published human 


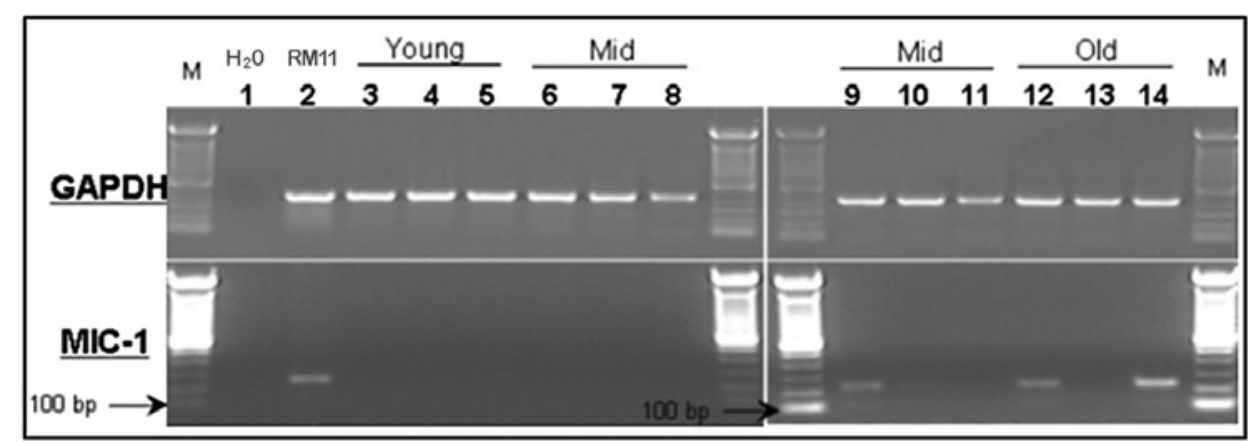

Figure 1. Expression of MIC-1 (lower panel) and GAPDH (upper panel) mRNA in different prostate lobes from prostate-specific antigen transgenic mice of different age groups. Lanes 3-5, young age; lanes 6-11, middle age; lanes 12-14, elderly. Lanes 3, 6, 9 and 12, dorsolateral prostate; lanes 4, 7, 10 and 13, ventral prostate; and lanes 5, 8, 11 and 14, anterior prostate. Lanes 1 and 2 are $\mathrm{H}_{2} \mathrm{O}$ and positive (RM11 mouse prostate tumor cells) controls, respectively.

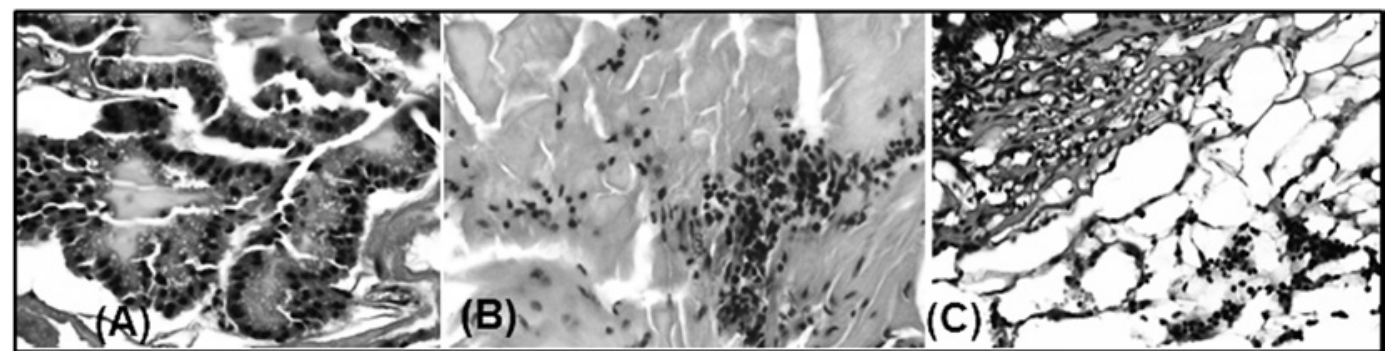

Figure 2. Representation of H\&E-stained slides of the formalin-fixed, paraffin-embedded prostate tissues from prostate-specific antigen-transgenic mice of different ages. (A) Representative example of normal prostate tissue harvested from a 4-week-old mouse with no evidence of inflammation. (B) and (C) Representative photomicrographs of prostate sections from a 15- and 24-month-old mouse, respectively, at different magnifications. An aggregate of chronic inflammatory cells is noted in (B), and in (C) diffuse chronic inflammation in the stroma (right upper corner) and glandular degeneration.

studies $(13,26,27), M I C-1$ gene expression was extremely low to non-detectable in the normal (healthy) prostate lobes of young (4 weeks) and middle-aged (13 months) mice (samples 3-8). However, higher MIC-1 levels were detectable in the prostate tissues of adult mice aged 15 months as well as those of 24-month-old elderly mice (samples 9-14).

Degree of inflammation (infiltrating cells) in the prostates of PSA-Tg mice. Prostate tissue samples from different age groups of PSA-Tg mice were further analyzed for the intensity of inflammation (analyzed by quantifying infiltrating cells in the tissue). Analysis of H\&E-stained slides showed that there was no infiltration of inflammatory cells in the prostate tissue samples harvested from the youngest mice (Fig. 2A). However, we found an increase in the intensity of inflammation in the samples harvested from 15-month-old mice (Fig. 2B). Furthermore, we noted a significant difference in the glandular architecture between samples from the different age groups. The prostates of the younger animals were healthy with normal-appearing glands that were organized in an orderly manner with no evidence of inflammation (Fig. 2A). By contrast, progressive degenerative glandular changes as well as an increase in inflammation were observed in the older mice, with the worst changes being detected in the oldest mice. Degeneration of epithelial lining and intraluminal sloughing of epithelial cells was extremely high along with a significant depletion of the prostatic secretion in the prostates of elderly PSA-Tg mice (Fig. 2C). Overall, the prostate tissue from 24-month-old elderly mice was severely damaged, possibly due to chronic inflammation; however, the examined slides did not reveal any sign of neoplasm. Thus, the presence of inflammation corresponds with the mRNA expression of the $M I C-1$ gene in the prostate tissues harvested from the PSA-Tg mice, confirming the hypothesis that an increase in the expression level of MIC-1 with increasing age may be due to inflammatory response. It has been reported that inflammation and aging influence the expression level of MIC-1 in correlation with infiltration of macrophages in the rat prostate (21).

Modulation of MIC-1 expression in a human prostate cancer cell line. To determine the possibility that $M I C-1$ gene expression may be regulated in response to inflammation at the early stage of cancer development, an androgen-responsive human prostate cancer cell line ( $\mathrm{LNCaP})$ was treated with various cytokines. Cytokine interleukin (IL)-1 $\beta$, IL-6, tumor necrosis factor- $\alpha$ (TNF- $\alpha$ ), and granulocyte macrophage colony-stimulating factor (GM-CSF) differentially regulate the expression of MIC-1 in LNCaP cells at the mRNA (Fig. 3A) and protein (Fig. 3B) levels. The selection of these cytokines was based on their association with inflammation. RT-PCR and western blot analysis showed consistency in the MIC-1 expression level following cytokine treatment. Western blot analysis revealed that two bands of approximately 40 and $15 \mathrm{kDa}$ correspond to pro-MIC-1 and mature MIC-1 protein (Fig. 3B). These predicted sizes of pro-MIC-1 and MIC-1 are consistent with previous studies $(9,22-24)$. Notably, our studies 


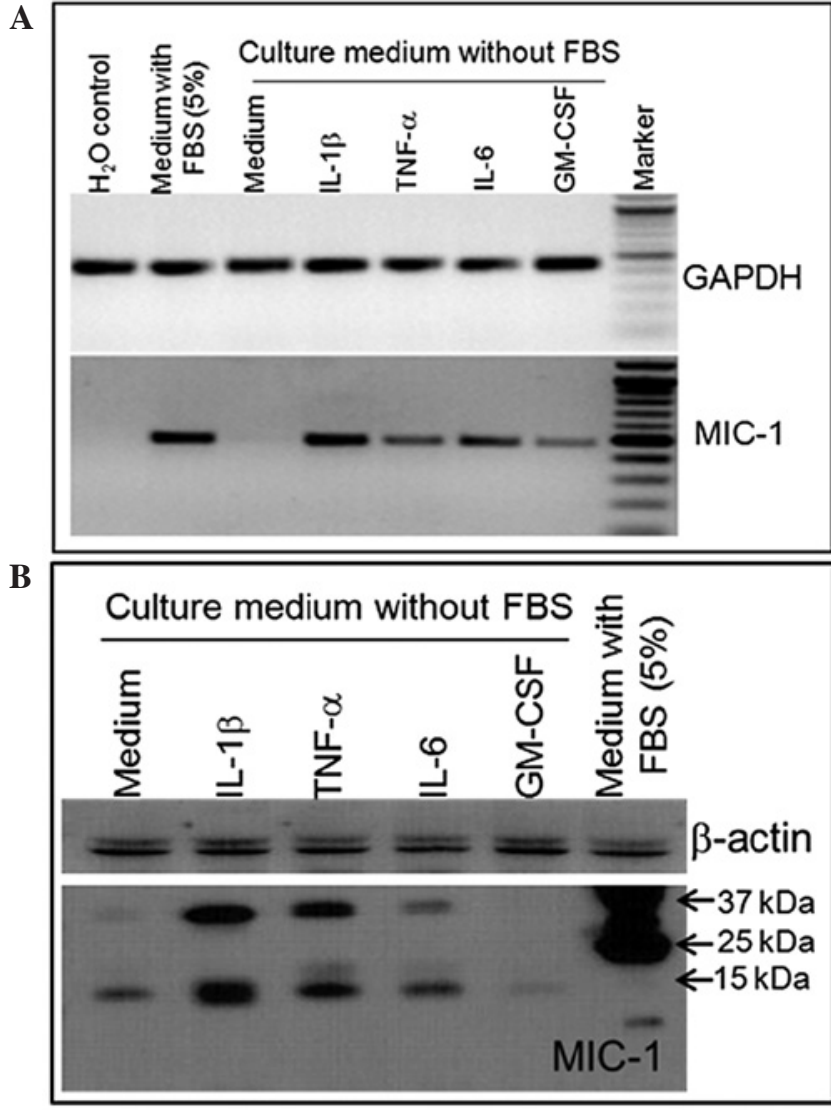

Figure 3. Cytokine-induced regulation of MIC-1 in human prostate cancer cell line (LNCaP cells). (A) RT-PCR analysis shows the expression of the $M I C-1$ gene on $\mathrm{LNCaP}$ cells treated with various cytokines. GAPDH was used as an internal control. (B) Differential secretion of the MIC-1 protein modulated by inflammatory cytokines. As an internal control, $\beta$-actin was used on the lysates from the same set of cells demonstrating the secretion of MIC-1 (lower panel). RPMI-1640 phenol red-free, serum-free medium served as a control for cytokine treatment, while the medium containing 5\% FBS served as a positive control.

revealed that IL-1 $\beta$ induction increased the expression of MIC-1 to the maximum level while the effect of GM-CSF was minimal in the LNCaP cell line. These results are supported by the observations that IL- $1 \beta$ is primarily secreted by macrophages which are crucial in regulating the inflammatory network in the host tissue. In macrophages, cytokines such as IL-1 $\beta$ and TNF- $\alpha$ induce the expression of MIC-1 (9). Such inflammation-induced MIC-1 production may function as an autocrine-paracrine regulator of MIC-1 in the host tissue, facilitating the process of enhanced cell proliferation $(9,25)$.

Most studies report on an increased expression of the $M I C-I$ gene in cancer and non-detectable levels in normal tissue when comparing matched prostate cancer and normal prostate specimens. Gene expression analysis between the normal peripheral zone (PZ) and transition zone (TZ) of the specimens obtained from prostate cancer patients revealed preferential expression of MIC-1 in the PZ (predominant site of tumor occurrence) compared to the TZ (site of benign prostatic hyperplasia) (26). The expression of MIC-1 is also reported in adult human prostate tissues with the existence of infiltrating lymphocytes in normal-appearing prostate tissues $(27,28)$. An induction in
MIC-1 gene expression in kidney, lung and liver tissue due to injury following surgical, chemical or heat shock methods has also been observed $(29,30)$.

In conclusion, this is the first study to show that the $M I C-I$ gene may be directly regulated by inflammation-associated cytokines in prostate cancer cells. Thus, activation of the $M I C-1$ gene may be an early response due to inflammation, infection or injury in the prostate for cell growth advantage leading to an environment favoring prostate cancer development. Since MIC-1 and macrophages are associated with inflammation, further studies are under way to investigate the mechanistic regulation of inflammation-induced MIC-1 gene in prostate cancer.

\section{References}

1. Jemal A, Siegel R, Xu J and Ward E: Cancer statistics, 2010. CA Cancer J Clin 60: 277-300, 2010.

2. Karan D, Thrasher JB and Lubaroff D: Prostate cancer: genes, environment, immunity and the use of immunotherapy. Prostate Cancer Prostatic Dis 11: 230-236, 2008.

3. Pienta KJ and Esper PS: Risk factors for prostate cancer. Ann Intern Med 118: 793-803, 1993

4. De Marzo AM, Platz EA, Sutcliffe S, et al: Inflammation in prostate carcinogenesis. Nat Rev Cancer 7: 256-269, 2007.

5. Platz EA and De Marzo AM: Epidemiology of inflammation and prostate cancer. J Urol 171: S36-S40, 2004.

6. Mantovani A, Allavena P, Sica A and Balkwill F: Cancer-related inflammation. Nature 454: 436-444, 2008.

7. Coussens LM and Werb Z: Inflammation and cancer. Nature 420: 860-867, 2002.

8. Wang D and Dubois RN: Prostaglandins and cancer. Gut 55: 115-122, 2006.

9. Bootcov MR, Bauskin AR, Valenzuela SM, et al: MIC-1, a novel macrophage inhibitory cytokine, is a divergent member of the TGF-beta superfamily. Proc Natl Acad Sci USA 94: 11514-11519, 1997.

10. Senapati S, Rachagani S, Chaudhary K, Johansson SL, Singh RK and Batra SK: Overexpression of macrophage inhibitory cytokine-1 induces metastasis of human prostate cancer cells through the FAK-RhoA signaling pathway. Oncogene 29: 1293-1302, 2010.

11. Breit SN, Johnen H, Cook AD, et al: The TGF-beta superfamily cytokine, MIC-1/GDF15: A pleotrophic cytokine with roles in inflammation, cancer and metabolism. Growth Factors 29: 187-195, 2011.

12. Cheung PK, Woolcock B, Adomat $\mathrm{H}$, et al: Protein profiling of microdissected prostate tissue links growth differentiation factor 15 to prostate carcinogenesis. Cancer Res 64: 5929-5933, 2004.

13. Karan D, Chen SJ, Johansson SL, et al: Dysregulated expression of MIC-1/PDF in human prostate tumor cells. Biochem Biophys Res Commun 305: 598-604, 2003.

14. Brown DA, Bauskin AR, Fairlie WD, et al: Antibody-based approach to high-volume genotyping for MIC-1 polymorphism. Biotechniques 33: 118-120, 122, 124 passim, 2002.

15. Brown DA, Moore J, Johnen H, et al: Serum macrophage inhibitory cytokine 1 in rheumatoid arthritis: a potential marker of erosive joint destruction. Arthritis Rheum 56: 753-764, 2007.

16. Tong S, Marjono B, Brown DA, et al: Serum concentrations of macrophage inhibitory cytokine 1 (MIC 1) as a predictor of miscarriage. Lancet 363: 129-130, 2004.

17. Brown DA, Lindmark F, Stattin P, et al: Macrophage inhibitory cytokine 1: a new prognostic marker in prostate cancer. Clin Cancer Res, 2009.

18. Sun J, Turner A, Xu J, Gronberg H and Isaacs W: Genetic variability in inflammation pathways and prostate cancer risk. Urol Oncol 25: 250-259, 2007.

19. Selander KS, Brown DA, Sequeiros GB, et al: Serum macrophage inhibitory cytokine-1 concentrations correlate with the presence of prostate cancer bone metastases. Cancer Epidemiol Biomarkers Prev 16: 532-537, 2007.

20. Karan D, Holzbeierlein J and Thrasher JB: Macrophage inhibitory cytokine-1: possible bridge molecule of inflammation and prostate cancer. Cancer Res 69: 2-5, 2009. 
21. Taniguchi S, Taoka R, Inui $\mathbf{M}$, Sugimoto $\mathbf{M}$ and Kakehi $\mathrm{Y}$ : Influence of inflammation and aging on macrophage inhibitory cytokine-1 gene expression in rat ventral prostate. Urology 73 : 410-414, 2009

22. Albertoni M, Shaw PH, Nozaki M, et al: Anoxia induces macrophage inhibitory cytokine-1 (MIC-1) in glioblastoma cells independently of p53 and HIF-1. Oncogene 21: 4212-4219, 2002.

23. Bauskin AR, Jiang L, Luo XW, Wu L, Brown DA and Breit SN: The TGF-beta superfamily cytokine MIC-1/GDF15: secretory mechanisms facilitate creation of latent stromal stores. J Interferon Cytokine Res 30: 389-397, 2010.

24. Bauskin AR, Zhang HP, Fairlie WD, et al: The propeptide of macrophage inhibitory cytokine (MIC-1), a TGF-beta superfamily member, acts as a quality control determinant for correctly folded MIC-1. Embo J 19: 2212-2220, 2000.

25. Chen SJ, Karan D, Johansson SL, et al: Prostate-derived factor as a paracrine and autocrine factor for the proliferation of androgen receptor-positive human prostate cancer cells. Prostate 67: $557-571,2007$.
26. Van der Heul-Nieuwenhuijsen L, Hendriksen PJ, van der Kwast TH and Jenster G: Gene expression profiling of the human prostate zones. BJU Int 98: 886-897, 2006.

27. Paralkar VM, Vail AL, Grasser WA, et al: Cloning and characterization of a novel member of the transforming growth factor-beta/bone morphogenetic protein family. J Biol Chem 273: 13760-13767, 1998.

28. Bostwick DG, de la Roza G, Dundore P, Corica FA and Iczkowski KA: Intraepithelial and stromal lymphocytes in the normal human prostate. Prostate 55: 187-193, 2003.

29. Zimmers TA, Jin X, Hsiao EC, McGrath SA, Esquela AF and Koniaris LG: Growth differentiation factor-15/macrophage inhibitory cytokine-1 induction after kidney and lung injury. Shock 23: 543-548, 2005.

30. Zimmers TA, Jin X, Hsiao EC, et al: Growth differentiation factor-15: induction in liver injury through p53 and tumor necrosis factor-independent mechanisms. J Surg Res 130: 45-51, 2006. 